International Journal of Bifurcation and Chaos, Vol. 12, No. 9 (2002) 2021-2029

(c) World Scientific Publishing Company

\title{
MATHEMATICAL MODELS FOR CELL-MATRIX INTERACTIONS DURING DERMAL WOUND HEALING
}

\author{
P. K. MAINI and L. OLSEN \\ Centre for Mathematical Biology, Mathematical Institute, \\ 24-29 St Giles', Oxford, OX1 3LB, UK \\ J. A. SHERRATT \\ Department of Mathematics, Riccarton, Heriot-Watt University, \\ Edinburgh, EH14 4AS, UK
}

Received November 11, 1998; Revised September 10, 2001

\begin{abstract}
This paper contains a review of our recent work on the mathematical modeling of cell interaction with extracellular matrix components during the process of dermal wound healing. The models are of partial differential equation type and allow us to investigate in detail how various mechanochemical effects may be responsible for certain wound healing disorders such as fibrocontractive and fibroproliferative diseases. We also present a model for wound healing angiogenesis. The latter has several features in common with angiogenesis during cancer tumour growth and spread so a deeper understanding of the phenomenon in the context of wound healing may also help in the treatment of certain cancers.
\end{abstract}

Keywords: Wound contraction; fibroproliferative disorders; angiogenesis.

\section{Introduction}

Recent advances in molecular and cellular biology have led to the rapid development of experimental research into the biochemical mechanisms underlying the processes of wound healing. Wound healing is an enormously complex dynamic spatiotemporal process and new insights are being gained by focussing on the interaction of specific processes involved for a particular aspect of healing. Mathematical modeling can play an important role by providing a theoretical framework within which these results can be analyzed, by exploring the potential of various proposed mechanisms to account for clinical observations, and suggesting novel biological mechanisms which may lead to new experimental approaches. Such models also provide experimentally testable predictions on the outcome of manipulating key biological parameters. In this respect, mathematical modeling may be thought of as another experimental tool.

In this paper we chose to focus on certain aspects of cell-matrix interactions during wound healing and review our recent work in this area. In Sec. 2 we consider a mechanochemical model framework for the contraction of wounds during normal healing. This is a complicated system of highly nonlinear equations and, in order to gain a greater understanding of the model, we consider caricature models within this framework that are more tractable and help us to investigate normal wound contraction (Sec. 3) and wound healing disorders, such as fibroproliferative diseases (Sec. 4). In Sec. 5 we consider the role of extracellular matrix in angiogenesis, the process by which a new vascular system is set up after wounding. 


\section{Mechanochemical Model Framework}

A crucial aspect of wound healing concerns the mechanical interaction of cells with their external environment. Cells deform and remodel the extracellular matrix (ECM) on which they move and ECM materials, in turn, affect cellular properties and cell orientation. Using the mechanochemical model framework developed by Murray and coworkers (see [Murray, 1993] for review, and [Murray et al., 1988; Murray \& Tranquillo, 1992]) we derived a mechanochemical model for dermal wound healing [Olsen et al., 1995]. We refer the reader to the original paper for full details, including experimental justification for each term within the model. The model consists of two cell types - fibroblasts and myofibroblasts, densities, $n$ and $m$, respectively; a generic growth factor, concentration $c$, and ECM, density $\rho$. These quantities obey the general conservation equation

$$
\frac{\partial Q}{\partial t}=-\nabla \cdot \mathbf{J}_{\mathbf{Q}}+f_{Q}
$$

where $Q$ is the quantity in question, the first term on the right-hand side models motion with flux $\mathbf{J}_{\mathbf{Q}}$, and the second term models production and degradation. To complete the model, a force balance equation is needed to account for the mechanical interaction of the cells with the ECM.

For simplicity, we present here the onedimensional version of the model, where $x$ is space and $t$ is time. The fibroblast cell equation takes the form:

$$
\begin{aligned}
\frac{\partial n}{\partial t}= & D_{n} \frac{\partial^{2} n}{\partial x^{2}}-\frac{\partial}{\partial x}\left[\chi(c, n) \frac{\partial c}{\partial x}+n \frac{\partial u}{\partial t}\right] \\
& +R(c) n\left(1-\frac{n}{K}\right)-\frac{k_{1} c n}{C_{k}+c}+k_{2} m-d_{n} n .
\end{aligned}
$$

Implicit in these equations is the assumption that there are three main factors contributing to cell flux: random diffusion, with constant diffusion coefficient, $D_{n}$; chemotaxis with chemotactic sensitivity $\chi(c, n)$, and advection in response to the displacement, $u(x, t)$, of the ECM. The four remaining terms on the right-hand side model cell kinetics and include logistic cell growth with linear rate enhanced by growth factor, fibroblast conversion to myofibroblast phenotype mediated by growth factor, conversion from myofibroblast back to fibroblast cell type, and cell death.
The myofibroblast equation takes the form

$$
\begin{aligned}
\frac{\partial m}{\partial t}= & \frac{\partial}{\partial x}\left[-m \frac{\partial u}{\partial t}\right]+\varepsilon_{r} R(c) m\left(1-\frac{m}{K}\right) \\
& +\frac{k_{1} c n}{C_{k}+c}-k_{2} m-d_{m} m .
\end{aligned}
$$

Here, we assume that the dominant contribution to myofibroblast flux is advection, and that mitosis takes the same form as that for fibroblasts, modulated by a constant scale factor $\varepsilon_{r}$.

The growth factor satisfies the equation

$$
\frac{\partial c}{\partial t}=D_{c} \frac{\partial^{2} c}{\partial x^{2}}+\frac{\partial}{\partial x}\left[-c \frac{\partial u}{\partial t}\right]+S(n, m, c)-d_{c} c .
$$

Implicit in this equation is the assumption that the dominant contributions to growth factor flux are random diffusion, with constant diffusion coefficient $D_{c}$, and advection. The remaining terms on the right-hand side model biosynthesis and degradation.

The ECM moves primarily by advection and satisfies the equation

$$
\frac{\partial \rho}{\partial t}=\frac{\partial}{\partial x}\left[-\rho \frac{\partial u}{\partial t}\right]+B(n, m . c, \rho),
$$

where $B(n, m, c, \rho)$ represents ECM biosynthesis and degradation.

Finally, modeling the ECM as a linear, isotropic, viscoelastic material, the displacement $u$ satisfies the force balance equation

$$
\mu \frac{\partial^{3} u}{\partial x^{2} \partial t}+E \frac{\partial^{2} u}{\partial x^{2}}+\frac{\partial \tau(n, \rho)}{\partial x}=F(\rho, u),
$$

where the first two terms on the left-hand side model viscous and elastic forces, respectively, and the third term models cell traction forces. These forces are balanced by the body forces $F(\rho, u)$. Note that this is very much a simplification as a more realistic model should include anisotropy and plasticity (see e.g. [Tracqui et al., 1995]).

The five equations, (2)-(6), with appropriate initial and boundary conditions (see below), constitute the mechanochemical model framework. Solving these equations in one spatial dimension is an approximation to "slash" wounds. Numerical simulations show that these equations admit solutions in which a traveling front of cell density moves into the wound, causing it to heal. Using biologically realistic forms for the functions $\chi(c, n), R(c), S(n, m, c)$, 


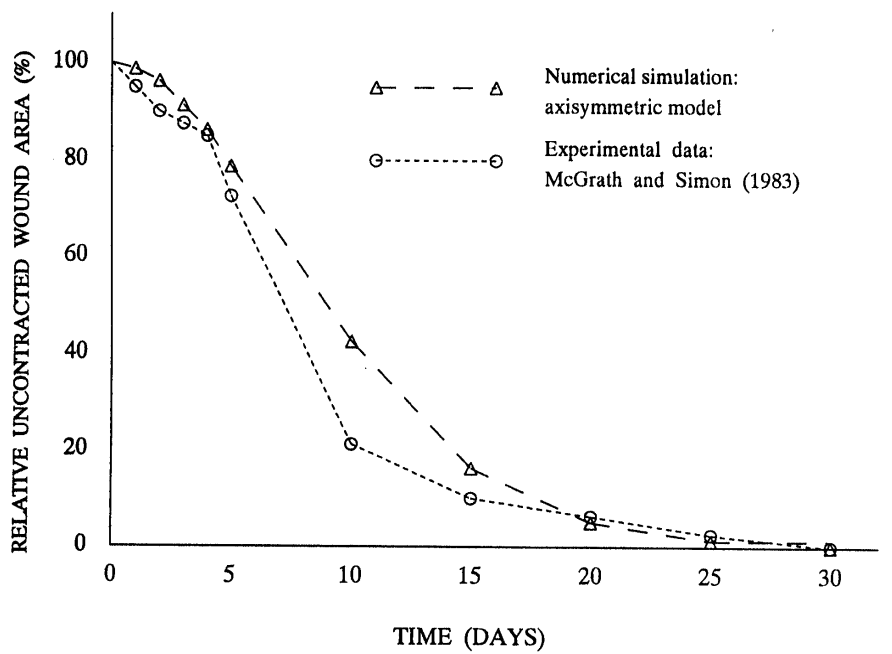

(a)

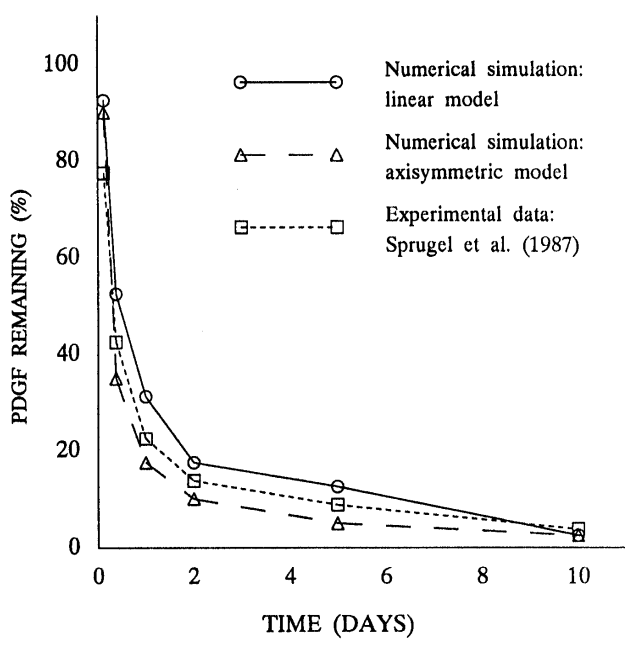

(b)

Fig. 1. Comparison of experimental data and numerical simulations of (2)-(6) for (a) wound contraction and (b) clearance of growth factor (PDGF - platelet-derived growth factor). See [Olsen et al., 1995], for full details.

$B(n, m . c, \rho), \tau(n, \rho), F(\rho, u)$, and estimates derived from experimental data for the parameters $D_{n}, D_{c}, K, k_{1}, k_{2}, C_{k}, d_{n}, d_{m}, d_{c}, \varepsilon_{r}, \mu$ and $E$, it can be shown that this model exhibits solutions for the decay of growth factor and rate of wound closure that closely agree with experimental results (see [Olsen et al., 1995], for full details and Fig. 1). Using a caricature model, we now proceed to analyze the possible contracted steady states exhibited by the model.

\section{Contracted Steady States}

To consider the potential of the model framework (2)-(6) to exhibit spatially-varying contracted steady states, Olsen et al. [1996] considered a simpler version of the model which focusses only on the mechanical aspects of the interaction. The nondimensionalized version of this caricature model takes the form

$$
\begin{gathered}
\frac{\partial n}{\partial t}=D_{n} \frac{\partial^{2} n}{\partial x^{2}}+\frac{\partial}{\partial x}\left[-n \frac{\partial u}{\partial t}\right]+n(1-n) \\
\frac{\partial \rho}{\partial t}=\frac{\partial}{\partial x}\left[-\rho \frac{\partial u}{\partial t}\right] \\
\mu \frac{\partial^{3} u}{\partial x^{2} \partial t}+E \frac{\partial^{2} u}{\partial x^{2}}+\frac{\partial \tau(n, \rho)}{\partial x}=F(\rho, u) .
\end{gathered}
$$

Here we consider only the fibroblast cell type and assume a simple form for logistic growth. We also assume in this model that there is negligible syn- thesis and degradation of ECM on the timescale of wound closure. This is a reasonable assumption to make in the stages prior to tissue remodeling during the process of wound healing.

By defining the initial wound space as $-1 \leq$ $x \leq 1$ and using symmetry at $x=0$ (the wound center), we may restrict attention to the semiinfinite domain $0 \leq x<\infty$. The boundary conditions are thus

$$
\frac{\partial n}{\partial x}(0, t)=\frac{\partial \rho}{\partial x}(0, t)=u(0, t)=0
$$

and

$$
n(\infty, t)=\rho(\infty, t)=1, \quad u(\infty, t)=0 .
$$

The initial conditions are

$$
\begin{gathered}
n(x, 0)=H(x-1), \\
\rho(x, 0)=\rho_{i}+\left(1-\rho_{i}\right) H(x-1), \\
u(x, 0)=0,
\end{gathered}
$$

where the initial ECM density $\rho_{i}$ inside the wound is due to the early, provisional wound matrix which is low in collagen and satisfies $0<\rho_{i}<1$, and $H(\cdot)$ is the Heaviside step function.

Consider now the healed steady state, $n=1$. Linearizing (8) about the initial profile, we have

$$
\rho \approx \begin{cases}\rho_{i}(1-\partial u / \partial x), & 0 \leq x<1 \\ 1-\partial u / \partial x, & x>1\end{cases}
$$


as suggested by the small-strain restriction (since the convective flux should be small). Substituting this into the steady-state equation for $u$ results in a second-order ordinary differential equation for $u$ which can be written in the (rescaled) form

$$
\begin{gathered}
u^{\prime}=v \\
v^{\prime}= \begin{cases}\frac{s \rho_{i} u(1-v)}{1-\rho_{i} \mathcal{T}\left[\rho_{i}(1-v)\right]}, & 0 \leq x<1 \\
\frac{s u(1-v)}{1-\mathcal{T}(1-v)}, & x>1,\end{cases}
\end{gathered}
$$

where $\mathcal{T}(\rho) \equiv \partial \tau(n, \rho) /\left.\partial \rho\right|_{n=1},{ }^{\prime}$ denotes ordinary derivative with respect to $x$, and $u$ satisfies the boundary conditions $u(0)=u(\infty)=0$. Here we have assumed that the body force, $F(\rho, u)$, is due to external tethering to the basement membrane and have modeled it by a linear spring, that is, $F(\rho, u)=s u \rho$, where $s$ is a constant.

Standard phase plane analysis of (11) and (12) shows that for $x>1$, the origin is a saddle (center) iff $\mathcal{T}(1)<1(>1)$. Linear stability analysis of the caricature model (7)-(9) shows that a necessary (but not sufficient) condition for the healed steady state to be stable is $\mathcal{T}(1)<1$ (see [Olsen et al., 1998]). As this must be the case for the model to be realistic biologically, we have that the origin of the ordinary differential equation system (11) and (12) is a saddle, and the boundary condition $u(\infty)=0$ implies that the solution must converge towards the origin along the stable manifold as $x$ tends to $\infty$. By tracing backwards in $x$ from infinity along the stable manifold, the solution reaches a point in the $\left(u, u^{\prime}\right)$-phase plane corresponding to $x=1$ where $u=u_{1}$, say. This must match the solution for $0 \leq x<1$.

Now, at the wound center, $u(0)=0$, but $v(0)$ is unspecified and is determined by matching to the "outer" solution at $x=1$. For $0 \leq x<1$, it can be shown that the origin can either be a saddle or a center, depending on the form of the function $\tau(n, \rho)$ and the values of the other parameters. If the origin is a saddle point, then the solution in $0 \leq x<1$ will be either monotonic increasing with increasing gradient or monotonic decreasing with decreasing gradient. If the origin is a center, then the solution in $0 \leq x<1$ may be oscillatory. Figure 2 illustrates the qualitative construction of such a solution and Fig. 3 illustrates various possible forms of steady-state solutions based on this construction. Modeling the traction term, $\tau(n, \rho)$, by $\tau(n, \rho)=\tau_{0} n \rho /\left(T^{2}+\rho^{2}\right)$, to account for the fact that traction forces depend on adhesion between cell surface receptors and binding sites on collagen fibres, but the ability of a cell to extend and retract protrusions within a collagen substrate is inhibited at relatively high collagen densities, where $\tau_{0}$ and $T$ are constant parameters, we have found, by numerical simulation, steady states for (11) and (12) of the forms illustrated in Figs. 3(a)-3(e).

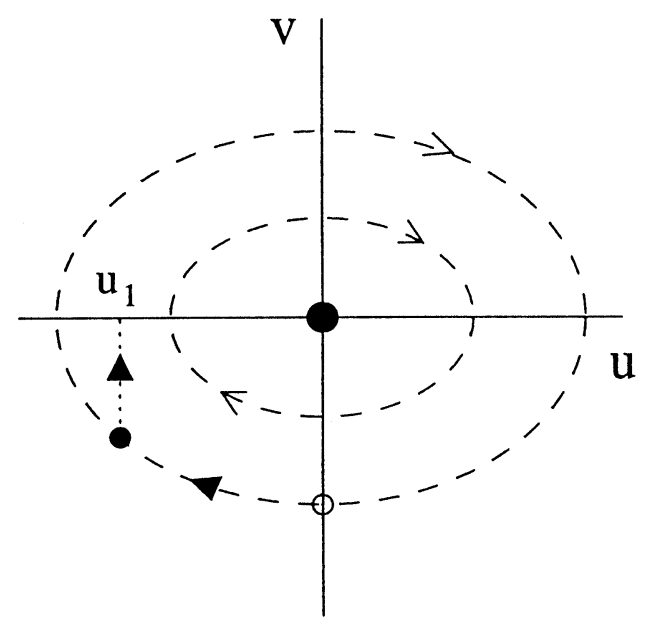

$$
0 \leq \mathrm{x}<1
$$

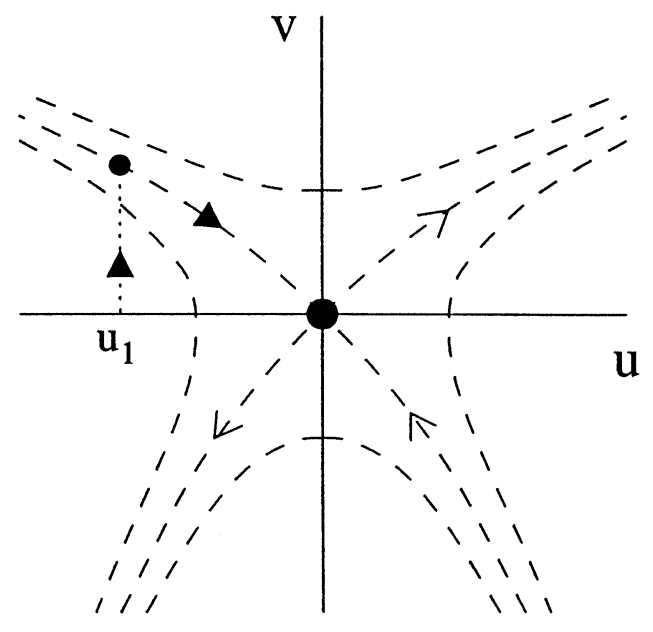

$\mathrm{x}>1$

Fig. 2. Qualitative illustration of a possible solution trajectory for the ODE system (11) and (12) for the case in which the origin is a center for $0 \leq x<1$ and a saddle point for $x>1$ with $u(x) \rightarrow 0$ from below as $x \rightarrow \infty$. [See also Fig. 3(b).] Dashed curves denote phase trajectories, with the contracted solution curve highlighted by solid arrows. 
(a)

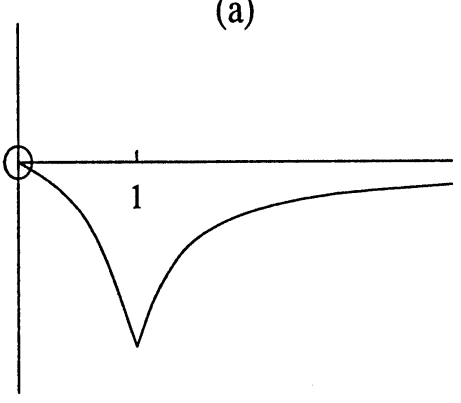

(d)

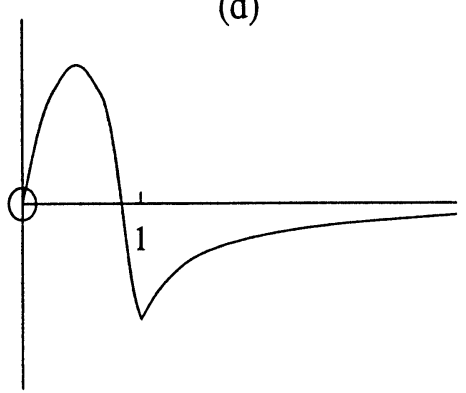

(b)

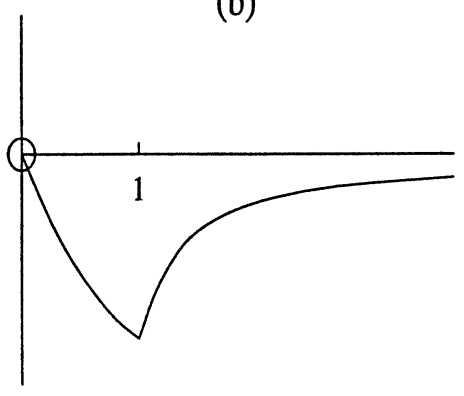

(e)

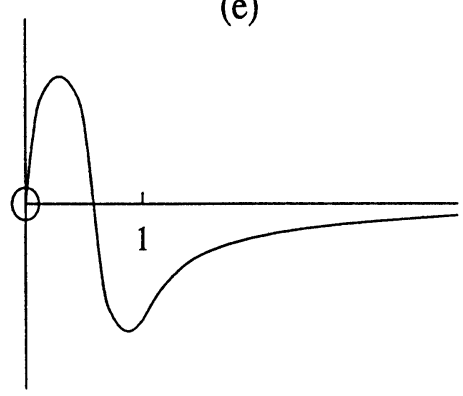

(c)

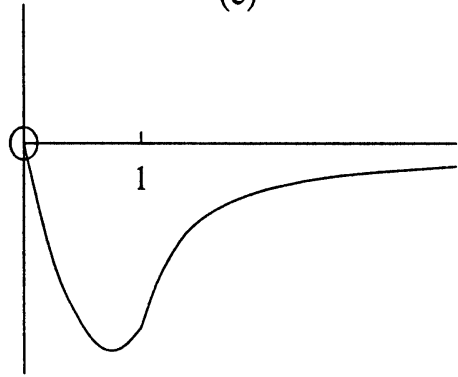

(f)

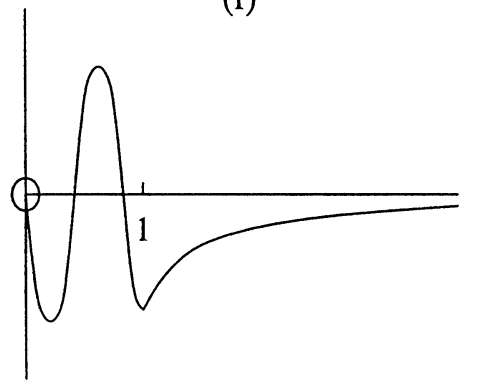

Fig. 3. Possible qualitative forms of the solution $u(x)$ to (11) and (12), representing contracted tissue displacement profiles. The point $\left(u, u^{\prime}\right)=(0,0)$ must be a saddle point for $x>1$ in the $\left(u, u^{\prime}\right)$-phase plane, with $u$ increasing to zero and $u^{\prime}$ decreasing to zero monotonically along the stable manifold in the top-left quadrant as $x \rightarrow \infty$. For $0 \leq x<1$, the origin may be either a saddle point, in which case the profiles for $u$ and $u^{\prime}$ are monotonic decreasing as shown in (a), or a center, in which case $u$ and $u^{\prime}$ oscillate about the origin as shown in (b-f); within this region, any number of oscillations is possible - e.g. (f) is equivalent to (b) modulo one period. Note that the above steady-state profiles, but with reversed signs of $u$ and $u^{\prime}$, are also admissible solutions of (11) and (12), representing expanded tissue displacement profiles since $u(1)$ would be positive. Recall that $x=1$ is the initial wound boundary.

Hence, this caricature model enables us to more fully understand the properties of the full model (2)-(6) and shows clearly that the model can exhibit spatially-varying contracted steady states, and is thus consistent with clinical observations on normal healing. For full details, see [Olsen et al., 1998].

\section{Fibroproliferative Wound Healing Disorders}

We now consider the application of the framework developed in Sec. 2 to fibroproliferative wound healing disorders. These disorders are characterized by the generation of abnormally large amounts of tissue during the healing process, leading to, for example, keloid scarring. Numerical simulations of the full model (2)-(6) show that it can exhibit solutions in which an excess of cells is observed, corresponding to a pathological state. To understand this more fully, we again consider a caricature model of the full system. In this case, however, we focus purely on the chemical aspects of the mechanochemical framework (2)-(6) by considering the cell-chemical submodel

$$
\begin{aligned}
\frac{\partial n}{\partial t}= & D_{n} \frac{\partial^{2} n}{\partial x^{2}}-\frac{\partial}{\partial x}\left[\chi(c, n) \frac{\partial c}{\partial n}\right] \\
& +\sigma\left[1+\frac{P c}{Q+c}\right] n\left(1-\frac{n}{K}\right)-d_{n} n \\
\frac{\partial c}{\partial t}= & D_{c} \frac{\partial^{2} c}{\partial x^{2}}+\frac{\kappa_{c} n c}{\gamma+c}-d_{c} c
\end{aligned}
$$

where $\chi(c, n)=\alpha /(\beta+c)^{2}$, and $\alpha, \beta, P, Q, \kappa_{c}$ and $\gamma$ are positive constants (see [Olsen et al., 1996] for full details).

This caricature model has two uniform steady states, $(n, c)=(0,0),(K, 0)$ corresponding, respectively, to the trivial, or nonhealing, state, and the normal dermal state. For appropriate parameter values, two other steady states exist which have both $n$ and $c$ nonzero, with $n>K$. These are the pathological, or diseased, steady states. Results from bifurcation analysis of (13) and (14), in the absence of diffusion, show that for a critical value, $\kappa_{c}^{1}$, 

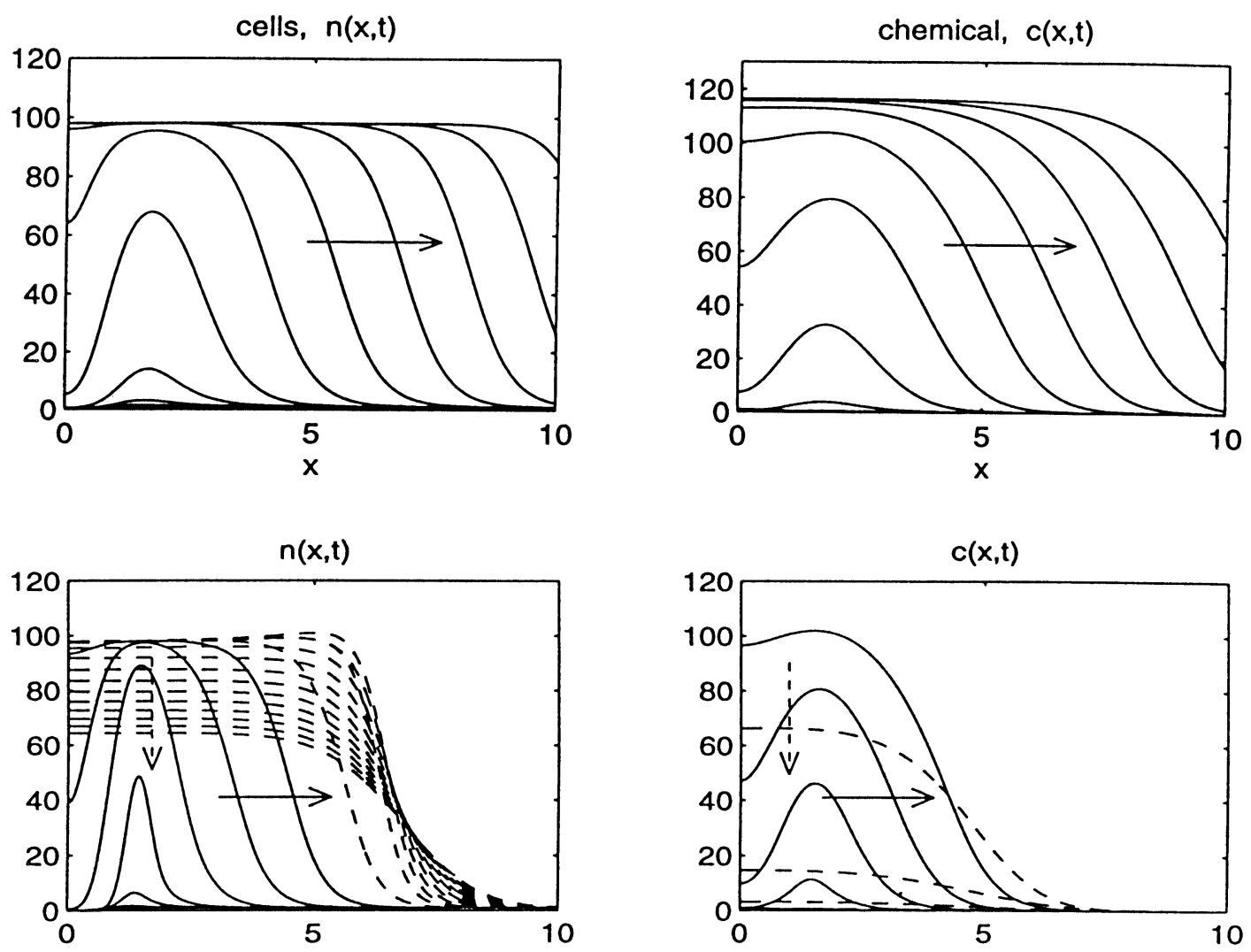

Fig. 4. Numerical simulations of (13) and (14) showing progression to pathological steady state (a), and cessation and regression for the case where $\kappa_{c}$ is reduced to zero after a certain time (b). See [Olsen et al., 1996] for parameter values.

of $\kappa_{c}$ (which can be found in terms of the other parameters) the dermal steady state remains locally stable but loses global stability as the pathological steady states appear. At $\kappa_{c}=\kappa_{c}^{2}$, the dermal steady state loses stability and the pathological state with higher cell density level becomes globally stable.

A traveling wave analysis of the model shows that trajectories from the dermal state to the pathological state are possible and a minimum wavespeed can be determined. Numerical simulations of the system show that such traveling waves do exist, but that reducing $\kappa_{c}$ can cause the waves to stop and to regress (see Fig. 4). This suggests that the reduction of the rate at which cells secrete growth factor can cause the disease to regress back to the normal dermal state. More detailed analysis of this model determines analytically how the bifurcation values of $\kappa_{c}$ depend on the other parameters in the model. In particular, the model exhibits hysteresis and therefore, counter-intuitively, $\kappa_{c}$ must be reduced considerably in order to progress from the diseased state to a healed state. This provides a clinically-testable method to help reduce this type of fibroproliferative disorder.

\section{A Mathematical Model for Angiogenesis}

Previous mathematical models for angiogensis have neglected the involvement of the ECM. This interaction has recently been considered by [Pettet et al., 1996] and [Olsen et al., 1997]. We briefly summarize the latter paper, which considers a model for cell-ECM interaction of the form:

$$
\begin{aligned}
& \frac{\partial n}{\partial t}+\overbrace{\frac{\partial}{\partial x}\left[C(m) n \frac{\partial m}{\partial x}-D(m) \frac{\partial n}{\partial x}\right]}^{\text {haptotaxis and haptokinesis }} \\
& =\overbrace{[A(m)-B n] n}^{\text {proliferation }} \equiv f(n, m) \\
& \text { production and } \\
& \frac{\partial m}{\partial t}=\overbrace{[P-Q m] n}^{\text {degradation }} \equiv g(n, m)
\end{aligned}
$$

where $n$ is endothelial cell density and $m$ is solidstate fibrillar ECM density. The model parameters 
are all non-negative. The haptotactic and haptokinetic coefficients are given, respectively, by $C(m)=$ $C_{0}\left(K_{C}+m\right)^{-2}$ and $D(m)=D_{0} m\left(K_{D}^{2}+m^{2}\right)^{-1}$. The cell proliferation rate is given by $A(m)=$ $A_{0} m\left(K_{A}^{2}+m^{2}\right)^{-1}$. We refer the reader to the original paper for the motivation and derivation of the above functional forms.

The model was considered on a one-dimensional domain to approximate "slash" wounds. To complete the model formulation, the following boundary and initial conditions are imposed: by symmetry, solutions need only be defined on the semi-infinite domain $x \geq 0$ subject to zero-flux at the wound centre, i.e. $C(m) n \partial m / \partial x=D(m) \partial n / \partial x$ at $x=0$; also, the cell and ECM variables remain at their normal dermal values far away from the wound, so $n \rightarrow n_{0}$ and $m \rightarrow m_{0}$ (say) as $x \rightarrow \infty$.
Initially, there are no endothelial cells and a low level of ECM (due to the early deposition of provisional matrix) inside the wound, so $n=0$ and $m=m_{\text {init }}$ (say) at $t=0$ for $0 \leq x \leq L$, with the unwounded values $n=n_{0}$ and $m=m_{0}$ outside the wound $(x>L)$. Note that $0<m_{\text {init }}<m_{0}$.

Investigation of the spatially uniform equilibria of this system reveals a continuum of unstable "acellular" states, in which $n=0$ and $m$ is unspecified, and a globally stable state, in which $n=A(m) / B$ and $m=P / Q$. These values are positive, and must be equal to $n_{0}$ and $m_{0}$, respectively, so this "dermal" steady state represents normal, unwounded tissue. Numerical simulations of the full system indicate that the cell and ECM profiles appear to evolve with approximately constant speed and form, after an initial transient and before the
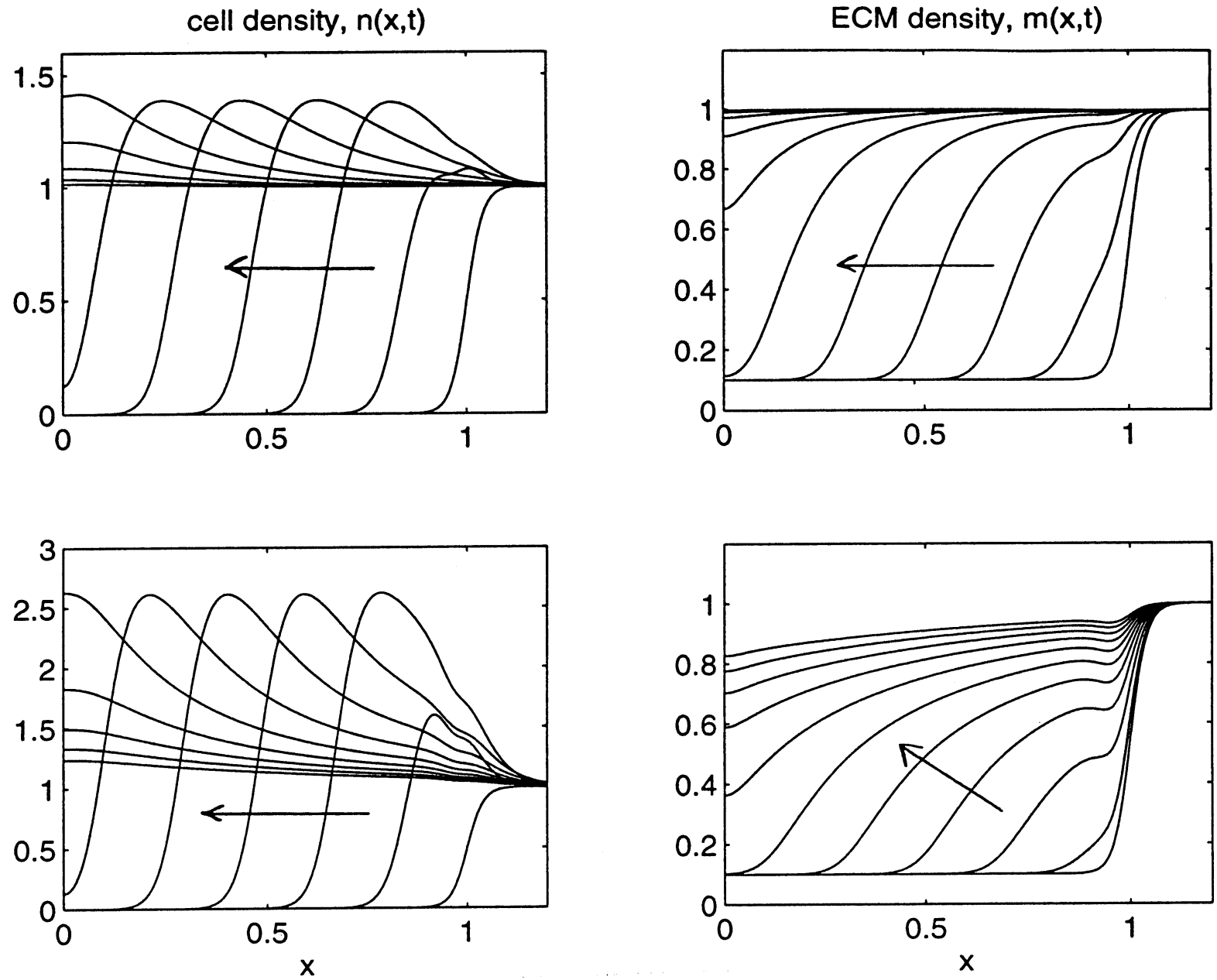

Fig. 5. Numerical solution of Eqs. (15) and (16) for different values of $\varepsilon$. Density profiles for cells $(n)$ and ECM $(m)$ are shown at time $t=0$ and at ten successive unit intervals. End-conditions as in the text. (Top row) parameter values are $D_{0}=0.005, \kappa_{D}=0.5, \chi_{0}=0.001, \kappa_{\chi}=0.5, \alpha_{0}=1.01, \kappa_{\alpha}=0.1, \beta=1, \varepsilon=1$ and $m_{\text {init }}=0.1$. (Bottom row) same, except $\varepsilon=0.2$, representing slower ECM kinetics. 
profiles reach the wound centre $(x=0)$. Cell density increases above its normal dermal value (unity) in the wave-front before gradually returning to its unwounded level, whereas the ECM profile is monotonic increasing. The gradients of the profiles are steeper in the wave-front than in the wave-back (see Fig. 5).

These phenomena simulate some important qualitative aspects of wound angiogenesis in vivo; in particular, the sharp increase in endothelial cell number prior to a gradual regression which parallels ECM accumulation.

This figure also illustrates that these features are more pronounced, and more realistic, when the ECM kinetics rate parameter $\varepsilon$ is lower. In wound healing, ECM remodeling occurs on a slower time scale than cellular proliferation, implying that this parameter should be relatively small in magnitude.

Traveling wave solutions can be investigated in the usual way and the following constraint on the wavespeed, $c$, can be derived:

$$
c \geq c_{\min }=2 \sqrt{D\left(m_{\text {init }}\right) \alpha\left(m_{\text {init }}\right)} .
$$

Moreover, the particular forms of the profiles can be exploited using perturbation analysis in the wavefront and wave-back to derive analytic approximations on how the profiles depend on the biological parameters that characterize ECM-regulation of wound angiogenesis (see [Olsen et al., 1997] for full details).

Numerical simulations of the two-dimensional version of the model show that angiogenesis occurs by cells and ECM moving inwards from both the wound margins and the wound base, the dominant effect being determined by the ratio of wound depth/breadth. Either parallel or perpendicular to the plane of the skin, the properties of the angiogenic process are very similar to those for the one-dimensional case, except for a narrow zone of interference between the orthogonally-propagating waves of cells and ECM.

\section{Discussion}

Wound healing in skin involves a complex sequence of inter-regulatory biological processes. To gain insight to the mechanisms underlying wound healing, we have chosen to develop models which focus on certain aspects of healing. The different models presented in this review focus on the mechanochemical aspects of cell-matrix interaction and illustrate how one can include key processes such as chemotaxis, chemokinesis and haptotaxis, as well as cell traction and matrix remodeling. This framework can be expanded to include cell alignment and orientation [Olsen et al., 1998]. The models capture key features of wound contraction, fibroproliferative diseases and wound angiogenesis and provide clinically testable predictions and treatments.

The production of different growth factors plays a crucial role in scar tissue formation and can also be modeled mathematically (see [Dale et al., 1996a, 1996b; Dale et al., 1997]). Collagen alignment also plays a crucial role in scar tissue formation. In normal tissue, collagen fibres are aligned in a crosslinked "basketweave" arrangement, whereas in scar tissue they are aligned more or less in the same direction. By considering a hybrid discretecontinuous model in which cells are represented as discrete entities while collagen and other key ECM components are modeled as continua we have shown how different cell-matrix interactions can combine to increase or decrease alignment [Dallon et al., 2000]. In particular, we have provided a possible explanation for anti-scarring therapies using transforming growth factor $\beta$.

This novel discrete-continuous hybrid approach has also been used to model certain phenomena occurring in tumour growth [Anderson et al., 2000]. Cancerous tumours share many common features with wound healing, for example, increased proliferation, and formation of new blood vessels [Anderson \& Chaplain, 1998]. The latter is thought to be crucial to the growth of many tumours and inhibiting angiogenesis using drugs at present provides one method of combating cancer growth (see e.g. [Folkman, 1985]). Hence, an understanding of these processes in wound healing may lead to important insights for the treatment of cancerous tumours.

\section{References}

Anderson, A. R. A. \& Chaplain, M. A. J. [1998] "Continuous and discrete mathematical models for tumourinduced angiogenesis," Bull. Math. Biol. 60, 857-899.

Anderson, A. R. A., Chaplain, M. A. J., Newman, E. L., Steele, R. J. C. \& Thompson, A. M. [2000] "Mathematical modelling of tumour invasion and metastasis," J. Theoret. Med. 2, 129-154.

Dale, P. D., Olsen, L., Maini, P. K. \& Sherratt, J. A. [1996a] "Travelling waves in wound healing," FORMA 10, 205-222. 
Dale, P. D., Maini, P. K. \& Sherratt, J. A. [1996b] "A mathematical model for collagen fibre formation during foetal and adult dermal wound healing," Proc. $R$. Soc. (London) B263, 653-660.

Dale, P. D., Maini, P. K. \& Sherratt, J. A. [1997] "The role of fibroblast migration in collagen fibre formation during foetal and adult dermal wound healing," Bull. Math. Biol. 59, 1077-1100.

Dallon, J., Sherratt, J. A., Maini, P. K. \& Ferguson, M. [2000] "Biological implications of a discrete mathematical model for disposition and alignment in dermal wound repair," IMA J. Math. Appl. Med. Biol. 17, 379-393.

Folkman, J. [1985] "Tumour angiogenesis," Adv. Cancer Res. 43, 175-203.

Murray, J. D., Maini, P. K. \& Tranquillo, R. T. [1988] "Mechanochemical models for generating biological pattern and form in development," Phys. Rep. 171, $59-84$.

Murray, J. D. \& Tranquillo, R. T. [1992] "Continuum of fibroblast-driven wound contraction: Inflammationmediation," J. Theoret. Biol. 158, 135-172.

Murray, J. D. [1993] Mathematical Biology (SpringerVerlag, Berlin).

Olsen, L., Sherratt, J. A. \& Maini, P. K. [1995] "A mechanochemical model for adult dermal wound con- traction and the permanence of the contracted tissue displacement profile," J. Theoret. Biol. 177, 113-128.

Olsen, L., Sherratt, J. A. \& Maini, P. K. [1996] "A mathematical model for fibroproliferative wound healing disorders," Bull. Math. Biol. 58, 787-808.

Olsen, L., Sherratt, J. A., Maini P. K. \& Arnold, F. [1997] "A mathematical model for the capillary endothelial cell-extracellular matrix interactions in wound-healing angiogenesis," IMA J. Math. Appl. Med. Biol. 14, 261-281.

Olsen, L., Maini, P. K., Sherratt, J. A. \& Marchant, B. [1998] "Simple modelling of extracellular matrix alignment in dermal wound healing. I. Cell flux induced alignment," J. Theoret. Med. 1, 175-192.

Olsen, L., Sherratt, J. A. \& Maini, P. K. [1998] "Spatially varying equilibria of mechanical models: Application to dermal wound contraction," Math. Biosci. 147, 113-129.

Pettet, G., Chaplain, M. A. J., McElwain, D. L. S. \& Byrne, H. M. [1996] "On the role of angiogenesis in wound healing," Proc. R. Soc. (London) B263, 1487-1493.

Tracqui, P., Woodward, D. E., Cruywagen, G. C., Cook, J. \& Murray, J. D. [1995] "A mechanical model for fibroblast-driven wound healing," J. Biol. Syst. 3, 1075-1085. 